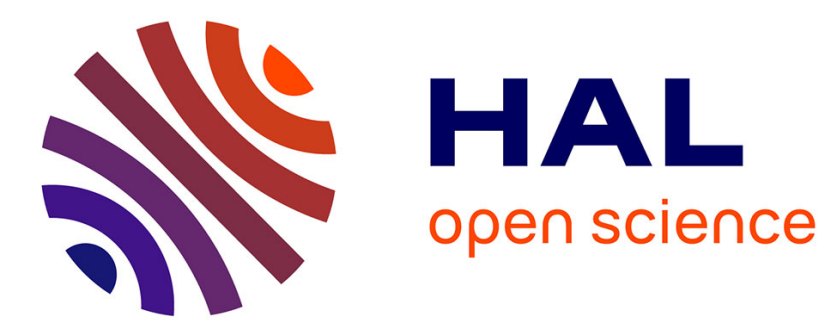

\title{
Fantasmagorie du musée : vers une visite numérique et récréative
}

\author{
Nicolas Navarro, Lise Renaud
}

\section{To cite this version:}

Nicolas Navarro, Lise Renaud. Fantasmagorie du musée : vers une visite numérique et récréative.

Culture et Musées, 2020, 35, pp.133-163. 10.4000/culturemusees.4713 . hal-03242439

\section{HAL Id: hal-03242439 \\ https://hal.science/hal-03242439}

Submitted on 31 May 2021

HAL is a multi-disciplinary open access archive for the deposit and dissemination of scientific research documents, whether they are published or not. The documents may come from teaching and research institutions in France or abroad, or from public or private research centers.
L'archive ouverte pluridisciplinaire HAL, est destinée au dépôt et à la diffusion de documents scientifiques de niveau recherche, publiés ou non, émanant des établissements d'enseignement et de recherche français ou étrangers, des laboratoires publics ou privés. 


\title{
(2) OpenEdition Journals
}

\section{Culture \& Musées}

Muséologie et recherches sur la culture

$35 \mid 2020$

Musées et mondes numériques

Dossier

\section{Fantasmagorie du musée : vers une visite numérique et récréative}

\author{
Phantasmagoria in museums: for digital and recreational tours \\ Fantasmagoría del museo: hacia una visita digital y recreativa \\ Nicolas NavarRo ET Lise RENAUd \\ p. $133-163$ \\ https://doi.org/10.4000/culturemusees.4713
}

\section{Résumés}

Français English Español

Dans un contexte de prolifération des applications mobile de visite de musées, cet article fait le choix d'interroger les discours qui escortent cette offre de médiation. Les vidéos de présentation en ligne de ces applications produites par les musées sont un terrain propice pour étudier les représentations d'une visite avec ces guides d'aide à l'interprétation. À partir d'une analyse socio-sémiotique d'un corpus de 23 vidéos de musées, cet article montre que ces vidéos travaillent à la rénovation de l'image de l'institution par un jeu d'affiliation à des pratiques de loisirs, tout en entraînant leur énonciateur dans le registre publicitaire. Il révèle l'élaboration d'une fantasmagorie qui puise ses valeurs et son esthétique dans les discours des industries de la communication et du numérique.

In the current context of the proliferation of mobile tour guide applications in museums, this article questions the discourses accompanying these outreach opportunities. Online videos presenting such applications produced by museums are a fertile terrain for the study of the representations of tours with these guides that aid interpretation. Thanks to a social and semiotic study of a selection of twenty three such videos produced by museums, this article demonstrates that these videos contribute to an actualization of a cultural institution's public image through its affiliation to leisure activities, while at the same time placing their production within the realm of advertising. It reveals the construction of a phantasmagorical representation whose values and aesthetic are rooted in the vocabulary of communications and digital industries.

En un contexto de proliferación de aplicaciones móviles para la visita de museos, este articulo opta por interrogar los discursos que acompañan esta oferta de mediación. Los videos de presentación online de estas aplicaciones producidas por los museos, son un terreno propicio para estudiar las representaciones de una visita con estas guías de ayuda a la interpretación. A partir de un análisis socio-semiótico de un corpus de veintitrés videos de museos, este artículo muestra que estos videos obran por la renovación de la imagen institucional a través de un juego de afiliación a prácticas recreativas, llevando a su enunciador hacia el registro publicitario. Revela la elaboración de una fantasmagoría que extrae sus valores y su estética en los discursos de las industrias de la comunicación y del mundo digital.

\section{Entrées d'index}

Mots-clés : application mobile, imaginaire, musée, médiation, discours promotionnels Keywords: mobile application, imagination, museums, outreach, promotional discourse Palabras clave: aplicación móvil, imaginación, museo, mediación, discursos promocionales

Notes de la rédaction

Manuscrit reçu le 10 avril 2019

Version révisée reçue le 20 décembre 2019

Article accepté pour publication le 3 mars 2020 


\section{Texte intégral}

1 Depuis une dizaine d'années maintenant, des applications mobiles de visite sont proposées aux publics des institutions culturelles et patrimoniales ${ }^{1}$. Bien qu'inscrites dans l'histoire longue des dispositifs portables d'aide à l'interprétation (dépliants, audioguides, visioguides), elles sont présentées dans la presse et les médias comme en mesure de modifier en profondeur l'expérience de visite. Elles participent de surcroît à un mouvement de densification de l'offre muséale d'aide à la visite, en complémentarité avec les signalétiques conceptuelles et panneaux scripto-visuels (Jacobi, 2016), les dispositifs multimédias (Schmitt \& Meyer-Chemenska, 2015) ou encore les visites guidées (Gellereau, 2005).

2 Les premiers travaux en muséologie qui portent sur ces applications pointent la pluralité des fonctions qui leur sont accordées en raison de la diversité des fonctionnalités techniques proposées. Ils définissent à la fois différents usages de l'application, mais aussi différentes pratiques de la visite muséale. En élaborant une typologie des propositions de médiations associées (fonctions " vitrine », " audioguide ", " médiation située », « visite virtuelle ", « catalogue », « événement »), une première étude transversale (Lesaffre et al., 2014) montre ainsi une potentialité de ces applications à regrouper et cumuler sur un même terminal l'ensemble de l'offre de médiation muséale. D'autres travaux scientifiques, s'appuyant sur des études d'évaluation de ces dispositifs, soulignent que l'offre d'applications, loin de se résumer à l'adjonction d'une nouvelle forme d'aide à l'interprétation, embarque avec elle des représentations sociales de la visite muséale et des imaginaires techniques liés aux outils numériques. Les analyses privilégiant la question d'un potentiel renouvellement de l'expérience de visite tendent à montrer que les applications mobiles constitueraient la visite muséale en un « hybride » entre pratiques culturelles et pratiques numériques (Dalbavie et al., $2016: 124)$. Les transformations espérées et effectives entrent en résonance avec le mouvement d'industrialisation des institutions et des biens culturels (Bouquillion et al., 2013) dont témoignent les stratégies de rénovation des musées engagés dans des logiques gestionnaires et communicationnelles (Tobelem, 2010). Dans ce contexte, les applications mobiles de visite apparaissent à la fois comme un produit d'appel permettant aux institutions de se situer dans l'agenda social, culturel et technique, mais également comme le signe d'une standardisation de l'offre de médiation muséale.

C'est pourquoi l'insertion de ces outils au musée est effectuée avec le renfort d'un discours promotionnel, discours d'accompagnement particulièrement dense sémiotiquement et hétérogène dans ces genres et formats (affiches publicitaires, communiqués de presse, rubriques de site web, etc.). L'un des effets de cette production discursive est de mettre en circulation des prescriptions d'usages. Elle construit donc autant une promesse de ce que serait une visite avec ces outils, qu'une « implication » diffusant des modèles de comportement à adopter (Jeanneret, 2014). Au-delà de la seule expression d'une stratégie commerciale telle qu'envisagée avec une approche par l'économie de l'attention (Citton, 2014 ; Martin-Juchat \& Staii, 2016), nous considérons que ce discours construit un archétype de la relation musée-public. Il représente des situations de visites et de médiations d'expositions, alimente l'horizon d'attente des visiteurs et conditionne leur réception de ces applications. Analyser ce discours permet donc d'identifier un modèle de représentation de la visite au musée considéré comme susceptible d'encourager l'usage de ces applications.

4 Dans l'ensemble du discours médiatisant ces applications, un registre discursif est récurrent : les vidéos promotionnelles. En effet, lors du lancement d'une application mobile de visite, les musées et leurs agences prestataires, afin de faire connaitre cette nouvelle offre de médiation, produisent et diffusent fréquemment des vidéos sur leurs propres sites internet et sur des plateformes de stockage (YouTube, Vimeo, Dailymotion). Cet outil de communication institutionnelle illustre le double mouvement de marchandisation et d'industrialisation à l'œuvre dans les institutions muséales (Regourd, 2012) : il vise la promotion d'un produit culturel spécifique à chaque musée tout en étant facilement reconnaissable par le public-consommateur.

5 Cet article présente les résultats d'une analyse socio-sémiotique de ces vidéos. Dans l'objectif de révéler les représentations sociales véhiculées, nous les considérons comme des énoncés dont nous analysons, à la manière du travail effectué sur les imaginaires du téléphone mobile par les chercheurs du Gripic (Jeanneret et al., 2005), à la fois les figures sémiotiques (composantes cinématographiques) et le contexte d'énonciation (titre, modalités de diffusion). Cette méthodologie nous permet d'identifier non seulement la fonction attribuée par le musée à ce discours, mais aussi les représentations circulantes d'une visite muséale avec ce type de guide.

6 Un corpus comportant 23 vidéos promotionnelles a été élaboré en veillant à l'hétérogénéité des musées représentés (musées d'art, d'histoire et de société, de sciences et techniques, qu'ils soient français ou étrangers) ${ }^{2}$. Cette hétérogénéité du corpus vise à répondre à la double hypothèse de l'expression de logiques de marchandisation par le recours à ces vidéos et de la construction d'un modèle de référence de la visite commun à tous les musées.

Dans un premier temps, cette étude souligne l'empreinte des industries de la communication et du numérique dans la construction de ce discours promotionnel. Elle montre ensuite comment la promesse puise dans l'imaginaire du numérique et les stéréotypes de la visite de musée pour valoriser l'offre de médiation. Enfin, elle interroge ce que ce discours fait à l’image du musée pour construire une relation méliorative à l'institution. Ce travail aboutit à mettre en lumière les rouages d'une fantasmagorie du musée, entendue comme un ensemble diffus et réactualisé de représentations de la visite muséale (Bonaccorsi, 2012b). 


\section{Des spots publicitaires pour la médiation muséale?}

Le contexte de diffusion des vidéos étudiées est symptomatique du rôle et du statut qui leur sont accordés par l'institution. Savoir ce que sont ces vidéos pour les musées renseigne sur la stratégie dans laquelle est inscrit leur déploiement. Comment peut-on qualifier ces vidéos ? À quoi servent-elles ? Et comment rendent-elles ce service?

\section{Entre communication commerciale et corporate}

Faisant la promotion d'une offre particulière à chaque musée, ces vidéos valorisent aussi l'institution. Elles relèvent d'une forme hybride de communication des organisations puisque centrée à la fois sur l'offre (communication commerciale) et l'organisation (communication corporate). Deux éléments permettent d'en rendre compte : leur intitulé et leur logique de diffusion.

10 Majoritairement, les musées de notre corpus ont choisi de nommer ces vidéos du nom de l'institution, suivi ou précédé des termes « application » ou « app »3, appellations qui ne correspondent pourtant pas au nom exact du logiciel sur les stores. Dans les magasins d'applications, le nom du programme est souvent confondu avec celui de l'institution : par exemple « Musée des beaux-arts de Lyon » ou « DMA » (Dallas Museum of Art) Le choix de dénomination des vidéos peut aussi être davantage centré sur la description du contenu offert par l'application, tout en conservant toujours une mention du nom de l'institution, comme pour «L'application MA VISITE du Musée d'arts de Nantes » ou encore l'« Application Mobile : Allen mène l'enquête au Musée de l'homme ». Ainsi, ces vidéos semblent davantage positionnées comme un moyen de montrer l'implication de l'institution muséale dans la mise en place d'une nouvelle offre de médiation que dans une stratégie visant à faciliter l'identification d'un produit culturel et donc sa consommation.

11 De façon plus occasionnelle, il est question de tutoriel, de teaser, voire même de l'expression d'une promesse de visite : "Une nouvelle façon de visiter le Muséum de Toulouse », "An app that will forever change how you enjoy museums » (SFMOMA : San Francisco Museum of Modern Art). En d'autres termes, les musées, à travers le choix de titre donné, d'une part insistent sur l'appartenance à l'institution - ce qui confirme le poids du rôle éditorial du musée dans la mise au point des applications de visite (Tardy, 2015) -, d'autre part expriment le caractère hybride de ces vidéos oscillant entre un statut de mode d'emploi et de publicité.

12 De surcroît, les modalités de diffusion de ces vidéos, le paratexte qui les escorte, soulignent aussi cette double affiliation de promotion et de mode d'emploi. Majoritairement, elles sont présentes dans une rubrique du site web du musée spécifiquement dédiée ou plus largement consacrée à l'offre de médiation. L'internaute peut activer des signes passeurs 4 lui permettant d'accéder aux téléchargements de l'application. Leur stockage sur les plateformes induit en outre que leur visualisation peut être effectuée directement via ces plateformes, et donc que les applications peuvent être consultées indépendamment d'une volonté de se renseigner sur les conditions d'une potentielle visite. Une dernière logique de diffusion identifiée consiste à les rendre consultables dans d'autres espaces éditoriaux en ligne du musée, un compte Facebook ou Twitter. Elles peuvent aussi être relayées dans des sites web d’institutions dont le contenu est centré sur l'actualité technologique du domaine culturel5. La circulation de la vidéo devient alors un moyen d'illustrer le caractère novateur du produit et, surtout, par métonymie, de l'institution elle-même, tout comme les effets de transformation de l'expérience muséale.

En somme, ces vidéos sont une forme spécifique de communication muséale, pleinement assumée par le musée, dont le propos embarque avec lui les représentations sociales du numérique et du numérique muséal. Le positionnement de ce discours par l'institution dans un rapprochement avec les genres discursifs des spots publicitaires et des tutoriels prouve que leur contenu et format doivent être en mesure à la fois de susciter du désir et de guider l’usage des applications mobiles de visite.

\section{Un format standardisé}

L'analyse de la composition de ces vidéos montre une certaine homogénéité de leur format. Leurs durées sont similaires, courtes, avec une majorité de musées privilégiant un format aux alentours d'une minute (en moyenne une 1 minute et 23 secondes), la plus courte faisant quinze secondes et la plus longue 4 minutes et 47 secondes. Cette durée est comparable à celle des vidéos de promotion d'entreprise ou de publicités institutionnelles. Elle est en revanche différente des formats de tutoriels ou de clips musicaux qui sont généralement plus longs, respectivement entre trois et cinq minutes.

Considérant leur composition sémiotique, on relève aisément une stratégie d'économie des signes mobilisés. Les composantes de l'expression cinématographique ne sont pas utilisées toutes ensemble. Les voix et bruits sont rares. Les mentions écrites encadrent généralement la vidéo et sont surtout présentes à la fin, tel un générique ou un pack shot (plan produit). La structure privilégiée est celle d'une succession de séquences comportant des plans qui s'enchaînent rapidement en coupe franche ou simple cut. La volonté de produire un effet de dynamisme est privilégiée. Les images mouvantes sont généralement liées et accompagnées par un son off, extra-diégétique, de type musique de fosse (Chion, 
2003). Deux logiques de choix musical sont présentes : soit la musique est d'ambiance de type électronique, assez dynamique ; soit elle est dépendante de la nature de la collection du musée : musique classique pour The Frick Collection (un concerto d'Albinoni), musique épique pour le National Military Museum de Soesterberg (Ultimate Sacrifice de Rusty Massive). D'un point de vue sémiotique, l'effet de sens repose donc soit sur une expression du dynamisme de la visite, soit sur une évocation sonore du contenu du musée.

On peut certes supposer que les logiques de diffusion sur les plateformes dédiées induisent d'adopter des formats courts qui accrochent l'attention. Toutefois, le format choisi positionne ces vidéos dans le registre publicitaire : le propos se doit d'être synthétique et immédiatement compréhensible, il doit dire l'essentiel en peu de temps. Ce parallèle avec la construction sémiotique des spots publicitaires confirme l'intérêt d'une analyse de ces vidéos et des représentations qu'elles véhiculent. La contrainte de format et de composition oblige à sélectionner les éléments montrés et à se concentrer sur le dispositif et son utilisation.

\section{Deux logiques énonciatives récurrentes héritées des équipementiers}

Une attention portée au contenu des plans et à leur structuration permet de relever une forme de normalisation voire de standardisation des modes de représentation. En effet, les images mouvantes des clips donnent à voir l'application à travers des extraits de son interface avec ou sans matérialité du terminal visible en usage (tablette ou téléphone), telle une démonstration de produit.

Dans cette rhétorique du mode d'emploi (Bonaccorsi, 2012a), deux logiques énonciatives sont adoptées. Dans certains cas, un terminal seul avec un contenu qui change est figuré en dehors de toute mise en contexte $^{6}$. Le contenu affiché se modifie sans que l'on aperçoive de mains le manipulant. La représentation est alors celle d'un objet à l'infinitif : la magnificence de l'objet repose sur une figuration hors de tout contexte spatiotemporel (Péninou, 1972).

Dans les autres vidéos, l'application est représentée en situation d'usage, c'est-à-dire en contexte de visite muséale (figure 2). Les plans alternent entre des figurations de l'espace muséal en plan général, des figurations d'objets de collection ou de visiteurs en déplacement en plan moyen, des plans rapprochés les montrant à l'arrêt, tenant un téléphone ou une tablette, puis des mains manipulant l'objet en gros plan. Les représentations des pratiques numériques reposent en ce sens principalement sur la portabilité et la tactilité des dispositifs et restent limitées à une mise en scène de mains agissant sur un écran. Comme constaté pour l'iconographie publicitaire de la lecture sur tablettes numériques, à travers l'esthétique de la gestualité, l'activité de la lecture numérique est ici aussi assimilée à une activité de visualisation (Bonaccorsi, 2012b). Les écrits d'écran sont vus plus que lus, ce qui tend à assimiler l'interprétation des objets muséaux à de la contemplation.

Figure 1. Présentation sous forme d'objet à l'infinitif.

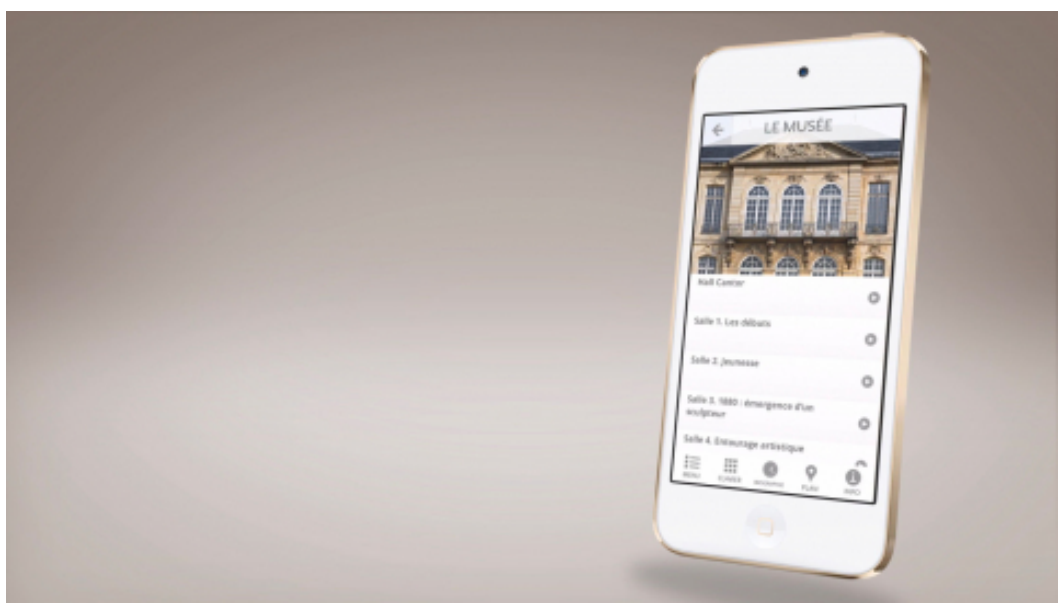

Application Musée Rodin. @ Musée Rodin.

Figure 2. Saynète de mise en usage. 


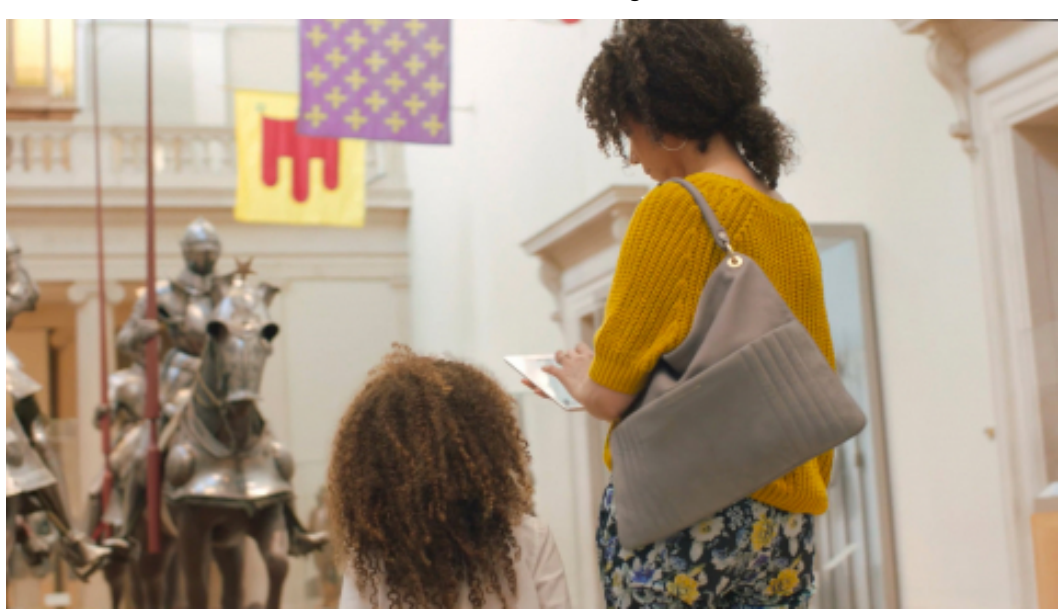

Application The MET. @ Metropolitan Museum of Art.

Quelle que soit la logique énonciative adoptée, la limite de l'écran de l'application représentée se confond rarement avec le cadre de la vidéo, comme s'il était nécessaire de conserver une distance plaçant le public en situation d'usage, voire contemplant une chorégraphie gestuelle (glisser, balayer, appuyer). Ainsi, l'argumentation repose sur une monstration du faire avec l'application, de sa mise en action, en gestes, en usage. Ce constat tend à indiquer que les musées font ici davantage la promotion d'une forme de visite muséale que d'une application dont ils sont les éditeurs.

21 Comme pour la durée et la composition, les deux logiques énonciatives identifiées sont similaires à celles utilisées par Apple pour promouvoir ses téléphones et tablettes mobiles depuis 2008. Cet équipementier, comme d'autres, oscille entre une monstration d'objet à l'infinitif et de saynètes de mise en usage (Renaud, 2016). Que cette proximité résulte de la circulation d'une imagerie, dépende des conditions de production (diversité des clients des agences, partenaires des projets) ou d'une inspiration assumée n'est pas ici primordiale. Cette similitude prouve surtout l'empreinte des industries de la communication dans le discours de l'institution muséale. Les musées produisent des spots (registre publicitaire) plus que des clips (registre du culturel) : la forme de leur discours les rapproche des industries de la communication plus que des industries culturelles. Le modèle suivi montre que ce qui prime est davantage la nature du dispositif technique (numérique) que la nature du contenu (culturel) (Navarro \& Renaud, 2019).

22 La reprise des formats, des codes et des modèles argumentatifs des publicités d'équipementiers indique que les musées ont bien du mal à s'extraire de cette vision de la culture numérique. En somme, l'introduction des applications mobiles de visite au musée n'a-t-elle pas au moins pour effet d'obliger le musée à traduire la visite muséale sous forme d'une représentation publicitaire reconnaissable par le plus grand nombre ? Quoi qu'il en soit, la mobilisation de cet imaginaire contraint indiscutablement la manière d'envisager et de représenter la visite au musée munie d'une application numérique.

\section{Une stéréotypisation de la visite numérique au musée}

Les vidéos mobilisent ainsi un format publicitaire, proche du spot, consécutif à l'arrivée d'un nouveau produit sur le «marché » de la médiation muséale. Elles font la promotion de cette modalité de médiation au musée qui se constitue en une « interaction médiatisée » (Jeanneret, 2014) entre le musée et son public. Par là même, ce discours promotionnel construit des représentations de la visite muséale en situation d'usage de ces applications. Qu'en est-il des représentations véhiculées par ces vidéos ? Quelles promesses de visite déploient-elles?

\section{Quand l'application éclipse les autres médiations}

En premier lieu, il convient de rappeler que les applications mobiles de visite sont mises en place au cœur d'un réseau déjà dense de médiations muséales. Pourtant, dans cette économie générale de la médiation, peu d'autres modalités sont évoquées dans les vidéos promotionnelles. Les plans ne font que très rarement apparaitre les panneaux de salles, cartels d'œuvres ou autres dispositifs scripto-visuels ou multimédias, et plus rares encore sont les visiteurs s'attardant à les lire ou les consulter. La visite sans application se résume alors à une rencontre avec l'objet de musée, en particulier avec l'œuvre d'art dans le cas des musées d'art, qui n'est pas suffisante pour activer le processus de reconnaissance (Veron, 1991). La représentation de cette visite souligne ainsi le manque que vient combler une nouvelle médiation muséale auprès de visiteurs paraissant parfois perdus dans le musée (MET : Metropolitan Museum of Art, New York) ou ne comprenant pas le sens des objets présentés devant eux (MANN : Musée d'archéologie nationale de Naples ; Muséum de Toulouse).

25 Dans ces vidéos, l'application de visite remplace, combine, voire éclipse tous les autres dispositifs jusqu'à les faire disparaître de l'écran. Cet effet est renforcé par la présentation, à la manière d'une 
démonstration produit, des fonctionnalités offertes par le dispositif. L'effet de catalogue de cet inventaire impacte à la fois la construction du discours promotionnel, où chaque séquence du film correspond à une fonctionnalité spécifique, mais également la représentation du dispositif comme couteau suisse pour la visite muséale. Cette idée est clairement exprimée dans la présentation de l'application du Canadian Museum for Human Rights : " The Canadian Museum for Human Rights mobile app contains a media-rich self-guided tour, interactive map, mood meter, augmented reality, and more. " Ainsi, bien que l'histoire des médias ait prouvé une absence de substitution lors de l'émergence d'un nouveau média (Charron, 2010), on voit activer ici l'argument d'une capacité de remplacement d'anciennes médiations par les outils numériques, ayant pour tendance de reléguer les autres dispositifs au rang d'objets dépassés et archaïques.

26 Une analyse des fonctionnalités représentées fait apparaître deux logiques. La première met en œuvre une diversification des fonctionnalités dans le temps, perceptible par l'année de sortie de l'application. Cette diversification peut sans doute s'expliquer par l'augmentation en nombre des technologies disponibles, mais aussi par des moyens plus importants attribués lors de leur conception. La seconde logique tend à montrer a contrario une limitation des fonctionnalités présentées, limitation potentiellement liée au format court des vidéos. Cette logique est sous-tendue également par les conditions et coûts de production des applications induisant une standardisation de leur structuration ; la promesse de facilité d'usage conduisant de manière récurrente à une sous-exploitation des fonctionnalités disponibles. Cette standardisation apparait également fortement impactée par la reproduction au sein de l'application de modalités de médiation préexistantes, en particulier la fonction d'audioguidage (Lesaffre et al., $2014: 13$ ).

27 Certaines fonctionnalités sont récurrentes dans les vidéos analysées et illustrent la construction archétypale d'une visite muséale avec application mobile. La première est celle de la diffusion de contenus de plusieurs natures et sous plusieurs formats. Ces contenus apportés sont à la fois des informations pratiques permettant l'organisation de la visite, mais surtout des informations et des savoirs permettant la reconnaissance des œuvres et du discours muséal. Qu'ils soient textuels (description des objets muséaux, panneaux), audio (sous forme de commentaires, d'extraits d'entretien avec des spécialistes) ou vidéo (numérisation haute définition, films), les contenus sont activés par le visiteur sur l'appareil, et l'application prend ici la forme assez classique d'un audioguide ou visioguide. Plus rarement, des animations $3 \mathrm{D}$ ou en réalité virtuelle proposent des reconstitutions des espaces muséaux ou des œuvres exposées (Rijksmuseum, Musée Grévin), voire une navigation au sein de cellesci (MANN). La possibilité d'une géolocalisation permanente au sein des espaces du musée s'ajoute alors à cette première fonctionnalité. Elle permet à la fois l'orientation du visiteur et le déclenchement spatialisé de contenus, lors d'une visite mettant en mouvement permanent les visiteurs au sein des salles du musée jusqu'à l'arrêt devant les œuvres à regarder. La vidéo du Rijksmuseum propose de suivre une visite préprogrammée grâce à l'application - les chefs-d'œuvre du musée - et suit pas à pas une visiteuse dans un parcours alternant déplacements les yeux rivés sur le téléphone pour se repérer et arrêts, après la reconnaissance des œuvres à contempler.

28 Se construit ainsi un archétype de la visite avec application caractérisé par une mobilité des visiteurs alternant des phases statiques devant les objets muséaux et des phases de déplacement. Les autres médiations muséales sont remplacées par la multiplicité des contenus apportés par l'application (textes, images, enregistrements, vidéos). Cette dernière constitue dès lors l'interface permanente entre le visiteur et l'espace muséal, et entre le visiteur et les objets.

\section{Promettre plus pour promettre mieux}

S'appuyant sur ce positionnement central de l'application dans la visite muséale, les vidéos construisent une promesse d'enrichissement de la visite, promesse fortement liée au discours circulant sur le numérique.

30 À un premier niveau, cette promesse s'inscrit dans les utopies de l'innovation liées au numérique, et parmi celles-ci, le gigantisme associé aux big data (Ibekwe-Sanjuan \& Paquienseguy, 2015). En effet, les contenus de l'application sont fréquemment caractérisés par leur diversité, et surtout par leur quantité7. Dans cette affiliation aux big data, la quantité des contenus est renforcée par leur qualité : haute définition des numérisations, haute technologie du dispositif.

31 Cette promesse s'inscrit alors de surcroît dans une volonté d'offrir un contenu adapté à chaque visiteur pour une meilleure compréhension du discours muséal. La diversité des contenus et des fonctionnalités entend répondre à la diversité du public. L'application satisfait des attentes multiples telles que mieux observer les œuvres et en découvrir des détails invisibles (Rijksmuseum), en apprendre plus (Guggenheim Museum), manipuler les objets muséaux (Airborne Museum). Les vidéos mêlent une promesse d'accessibilité au savoir - grâce à un contenu complémentaire aux éléments présents dans les salles - à une promesse d'expérience, d'un rapport sensible aux œuvres. La focalisation sur les attentes supposées des visiteurs est à la fois révélatrice du tournant communicationnel des musées depuis la nouvelle muséologie, mais aussi des discours portés sur le numérique. Renforcés par le mouvement du marketing expérientiel, ces discours ont fait des dispositifs des amplificateurs émotionnels qui jouent sur les affects et les perceptions du monde environnant (Renaud, 2016).

32 L'expérience des utilisateurs dépasse alors la seule réception des contenus culturels apportés par les applications pour également prendre en compte leurs déplacements dans l'espace muséal. L'application entend ainsi résoudre des difficultés d'orientation par une promesse de fluidité et de mobilité justifiée 
par le déploiement de la géolocalisation. Elle permet au visiteur de toujours se repérer ${ }^{8}$, grâce notamment à des plans dits "interactifs » (Musée Rodin), tout en optimisant la rencontre avec les objets muséaux. L'application condense les différents niveaux de signalétique (Jacobi \& Le Roy, 2013) à la fois directionnelle, en permettant d'orchestrer les déplacements d'un point à un autre, et conceptuelle, en positionnant le visiteur de manière optimale face à l'œuvre pour une meilleure réception des contenus culturels.

33 Les vidéos construisent ainsi une promesse de visite avec application caractérisée par son efficacité, comme une réponse permanente aux attentes du visiteur, tant préalables que pendant sa visite. Impossible de se perdre, de ne pas comprendre, toujours une expérience nouvelle en sont les leitmotivs. Pourtant, si cette promesse prolonge les enjeux propres aux médiations muséales, dont elle reprend en partie certains codes, elle démontre surtout le poids prégnant des enjeux du numérique. Les comportements des visiteurs illustrent une focalisation sur l'écran du téléphone et de la tablette, qui semble faire « écran » à la médiation (Navarro \& Renaud, 2019).

\section{Vers une pratique récréative de la visite au musée}

Plus qu'une valorisation de l'offre de médiation, les vidéos véhiculent un discours promotionnel visant une transformation de l'image du musée et de la relation qu'il instaure avec le visiteur, comme l'illustre le choix du titre des applications évoqué en première partie. À travers leurs vidéos, les musées présentent ce guide numérique comme le moyen de se départir d'un ensemble de clichés négatifs sur la visite muséale (incompréhension, élitisme, ennui, etc.). Cette stratégie discursive est-elle envisagée comme celle qu'il faut adopter pour séduire non seulement les publics des musées mais aussi les audiences des plateformes de vidéos ? Comment se matérialise-t-elle?

35 La visite mise en scène repose sur les codes d'une pratique culturelle classique (une sorte de tutoriel à la visite muséale), facilement reconnaissable, tout en s'hybridant avec des pratiques de loisir. Il en ressort un modèle relationnel musée-visiteur individualisé dont la satisfaction est représentée comme inhérente à la manipulation de l'écran.

\section{Une visite standard dans un musée indifférencié}

L'analyse des logiques de représentation des vidéos permet de constater un effet de standardisation de la figuration de la visite muséale. Elle est tellement similaire d'une vidéo à l'autre qu'elle semble ne pas dépendre du lieu dans lequel elle prend forme. Le scénario type montre un visiteur entrant dans un musée, démarrant son application puis marchant dans les salles d'exposition jusqu'à sa sortie de l'institution'.

37 Le musée est en ce sens présenté comme une institution générique dans lequel se déroule une pratique numérique, du fait de l'outil mobilisé, et culturelle, du fait du lieu où elle prend place. En effet, le musée n'est pas toujours reconnaissable : sa représentation visuelle peut être totalement absente (vidéo valorisant l'objet à l'infinitif) ou évoquée brièvement ${ }^{10}$. L'identification du musée se limite fréquemment à la figuration de son architecture extérieure, d'une salle ou d'un objet de collection emblématique, tels la collection d'armures ou le temple de Dendur au MET.

Par ailleurs, le musée représenté est un lieu où les autres visiteurs sont rares ou assimilés au décor. La visite seule est la modalité principale représentée. Elle peut parfois s'effectuer en groupe (couple, famille), mais toujours avec une limitation de la présence des visiteurs non membres de ce groupe. Il n'y a jamais de flux importants visibles, même dans des musées où l'ultra-fréquentation est avérée. L'échelle serrée des plans utilisée conduit d'ailleurs souvent à ne pas rendre perceptible l'espace environnant le visiteur et potentiellement occupé par d'autres publics. Il en ressort une figuration de la relation construite avec l’institution avant tout individuelle et exclusive (figures 3 \& 4).

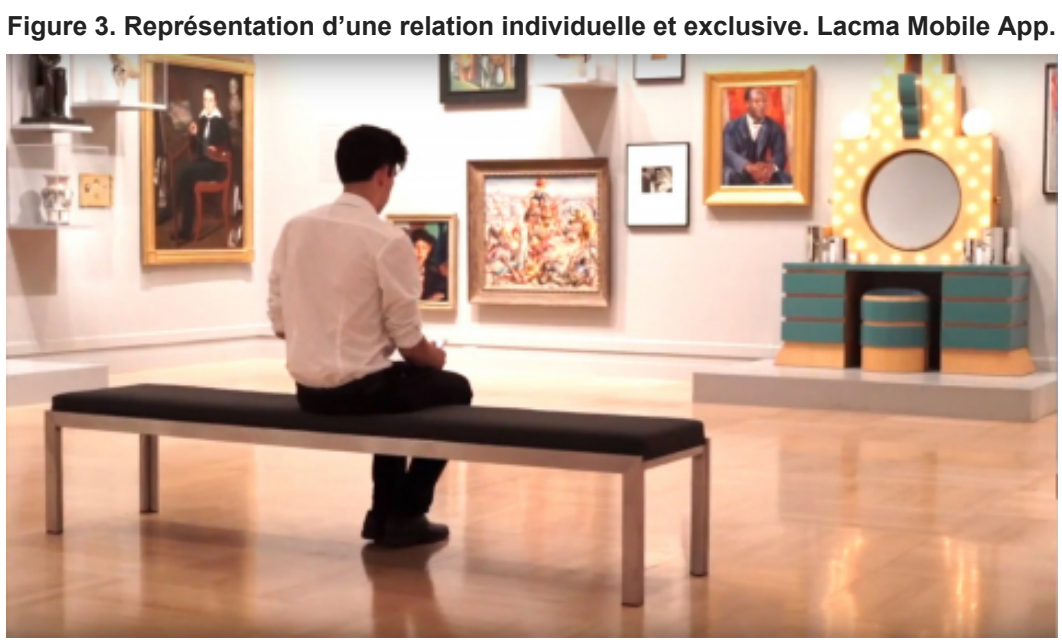


Figure 4. Représentation d'une relation individuelle et exclusive. Application Musée d'arts de Nantes.

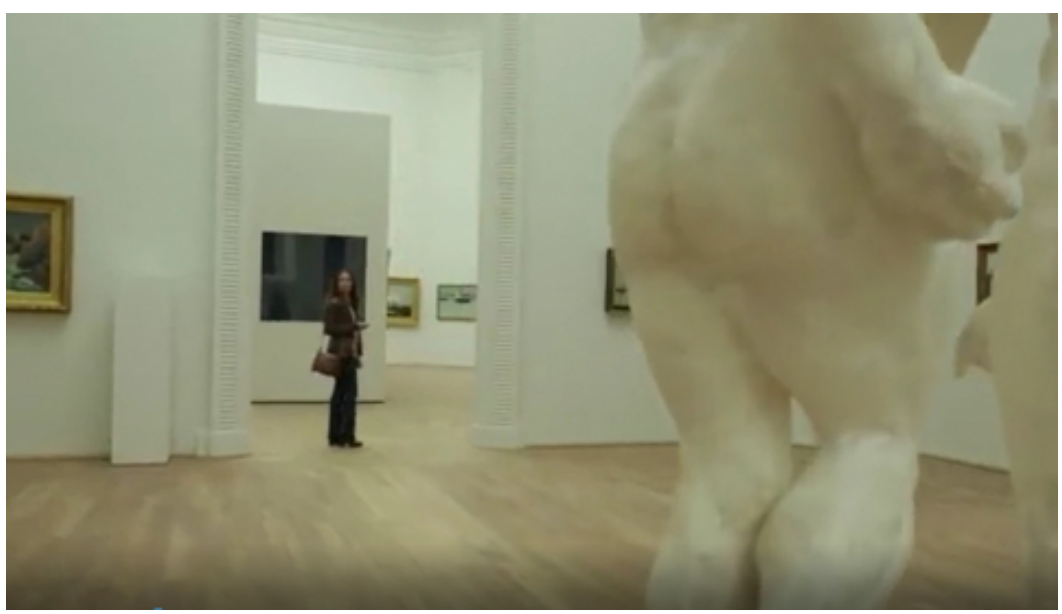

() Musée d'arts de Nantes.

Quant au visiteur représenté, sa figuration en fait un « utilisateur générique ». La parole lui est rarement donnée ${ }^{11}$, et son rôle est limité à montrer comment fonctionne le dispositif. C'est avant tout un démonstrateur. Pour autant, si l'argumentation peut exprimer l'idée d'une accessibilité pour tous les visiteurs ${ }^{12}$ et qu'au premier abord le visiteur semble " un individu lambda », l'analyse de ses attributs (critère d'habillement, d'âge, de sexe, etc.) indique qu'il correspond à un stéréotype plus largement valorisé dans la publicité (classes supérieures, classes créatives, trentenaire, urbain).

On peut s'interroger sur les causes de cette standardisation. Est-ce le format court qui conduit à produire cette figuration ? Est-ce la volonté de s'adresser à un public élargi qui induit une représentation de la visite comme une suite d'activités reproductibles d'un lieu à un autre, mécaniques et valables pour tous (se déplacer, s'arrêter, regarder...) ? Est-ce en raison des caractéristiques techniques du terminal (portabilité, écran, écouteurs) qu'est diffusée l'image d'une relation individualisée à l'institution?

Quoi qu'il en soit, il en ressort une représentation assez sommaire en termes d'actions des visiteurs et plutôt traditionnelle de la visite de musée, représentation en contradiction avec les études menées depuis plusieurs décennies en muséologie (Eidelman et al., 2013). Munie d'une application mobile, la visite devient une activité qui peut se dérouler dans n’importe quel musée.

\section{Une pratique de loisir}

Si le modèle comportemental représenté ne met pas au jour un bouleversement des relations visiteurmusée - bien au contraire il les limite à quelques actions -, les transformations envisagées de la relation musée-public sont situées sur un autre plan. C'est l'image même de la visite de musée, en tant que pratique culturelle supposée élitiste, qui est présentée comme modifiée. Dans notre corpus, elle est affiliée à une visite touristique ou de loisir qu'un public peut effectuer dans son quotidien au même titre que d'autres pratiques culturelles ou de divertissement.

Cette affiliation passe ainsi par une extension du rapport au musée dépassant la seule visite in situ. Les vidéos représentent une relation qui débute en dehors du musée, lors de préparatifs initiés dans des lieux publics (café-restaurant, bibliothèque, jardins publics), et se prolonge après avoir quitté l'espace muséal, par exemple par la consultation et la transmission de photographies de sa visite (figures 5 \& 6). La relation au musée déborde donc l'espace-temps de la visite pour s'exprimer dans d'autres lieux, et ce, jusqu'au domicile des visiteurs ${ }^{13}$, tout en s'intégrant dans une temporalité élargie grâce à l'accès permanent à des œuvres numérisées et des visites virtuelles des espaces d'exposition ${ }^{14}$. La relation représentée, et ainsi construite, revendique une forme de proximité, voire d'intimité entre le musée et son public.

Figure 5. Préparatif d'avant-visite. 


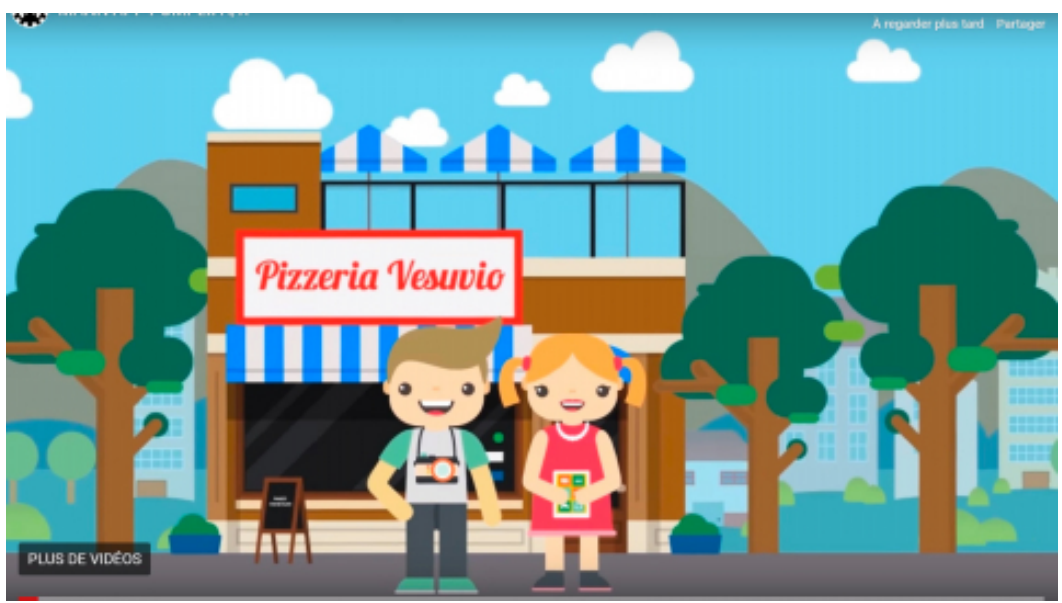

Mann App. (C) Musée d'archéologie nationale de Naples.

Figure 6. Prolongation d'après-visite.

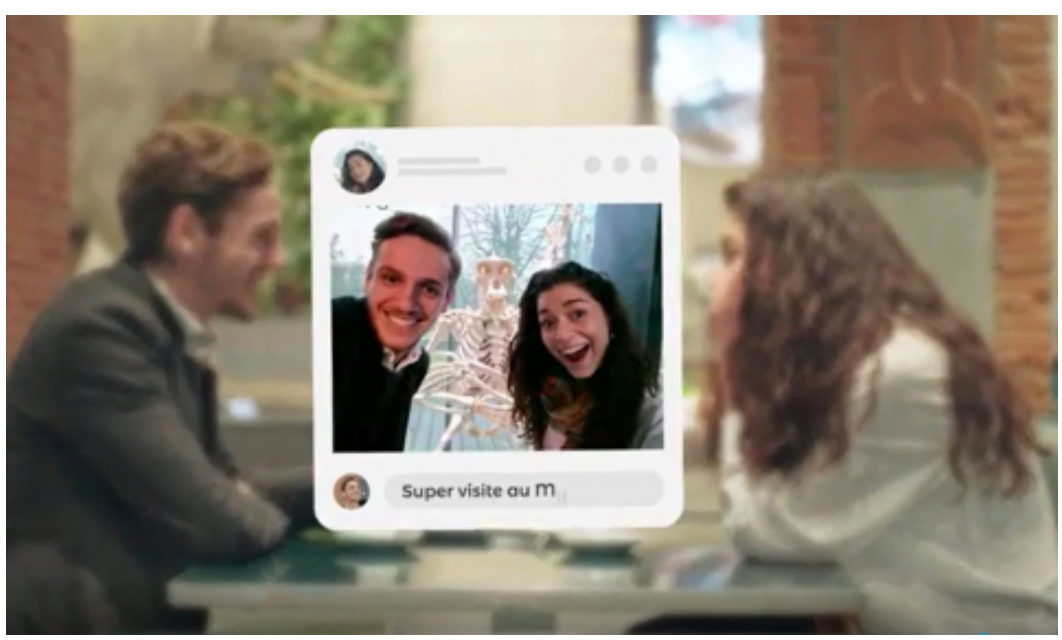

Visite museum Mobile. ( ) Muséum de Toulouse.

Ce rapport intime est renforcé par une individualisation fréquente et assumée de la relation au musée ${ }^{15}$. L'application en démonstration devient un outil pour construire, plus qu'une visite personnalisée, une appropriation personnelle de l'institution : "Find what's you're looking for - your way, on your own time »(DMA). La socialisation au musée, par des visites à plusieurs et des interactions entre visiteurs, peu présente, est mentionnée dans le discours argumentatif plus que véritablement illustrée dans les vidéos ${ }^{16}$. L'échange s'effectue plutôt à la suite de la visite par la mise en scène d'un partage photographique au sein des réseaux socionumériques, encouragé et sollicité par l'application ${ }^{17}$, comme une manière de faire parler les visiteurs, d'engager le dialogue avec eux, mais surtout comme une réappropriation par les institutions du discours des industries de la communication et des industries du web dit « social » (Escande-Gauquié \& Jeanne-Perrier, 2017).

Cette diffraction entre le temps de la reconnaissance du discours muséal, celui de l'expérience de visite, et le temps de son partage, celui de la discussion, illustre selon nous une tendance à la représentation du rapport au musée via l'utilisation de l'application comme une pratique de loisir s'appuyant sur des dimensions ludiques et hédonistes. En effet, certaines fonctionnalités s'inscrivent dans un processus de gamification du musée en proposant des chasses au trésor ou des recherches aléatoires dans les collections ${ }^{18}$, jusqu'à des formes narratives proches du jeu vidéo. Ces dernières, développées de manière privilégiée dans des musées traitant d'histoire naturelle (Musée de l'homme) ou des conflits armés (Airborne Museum, National Military Museum), s'inspirent des tendances fortes de l'industrie du jeu vidéo. Mais en plus des seules fonctionnalités, ce sont aussi les mises en scène des individus dans ces vidéos qui véhiculent cette dimension ludique : le visiteur parle aux objets du musée (Rijksmuseum), se prend en photo dans les mêmes poses que les œuvres (DMA, Muséum de Toulouse) (figures 7 \& 8). Le rapport au musée ainsi représenté apparait décontracté et récréatif. La délectation face aux œuvres est schématiquement représentée par des visiteurs quasi extatiques : gros plans sur des visages souriants ou exprimant la satisfaction, interjection lors de la reconnaissance d'une œuvre.

Figure 7. Mise en scène d'une visiteuse parlant aux objets. 


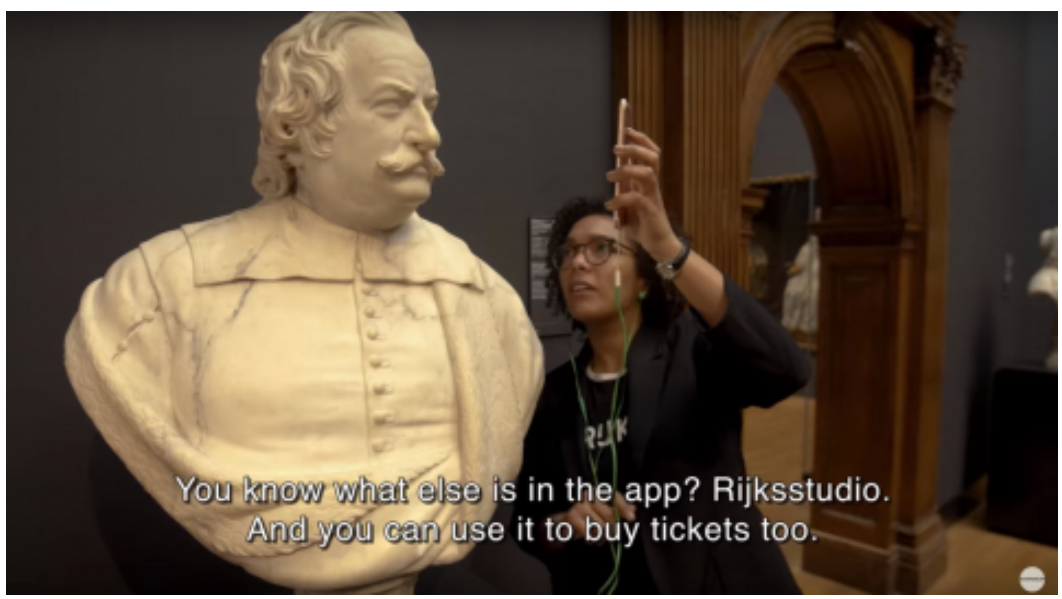

Rijksmuseum App. @ Rijksmuseum.

Figure 8. Mise en scène d'une visiteuse se prenant en selfie devant une œuvre.

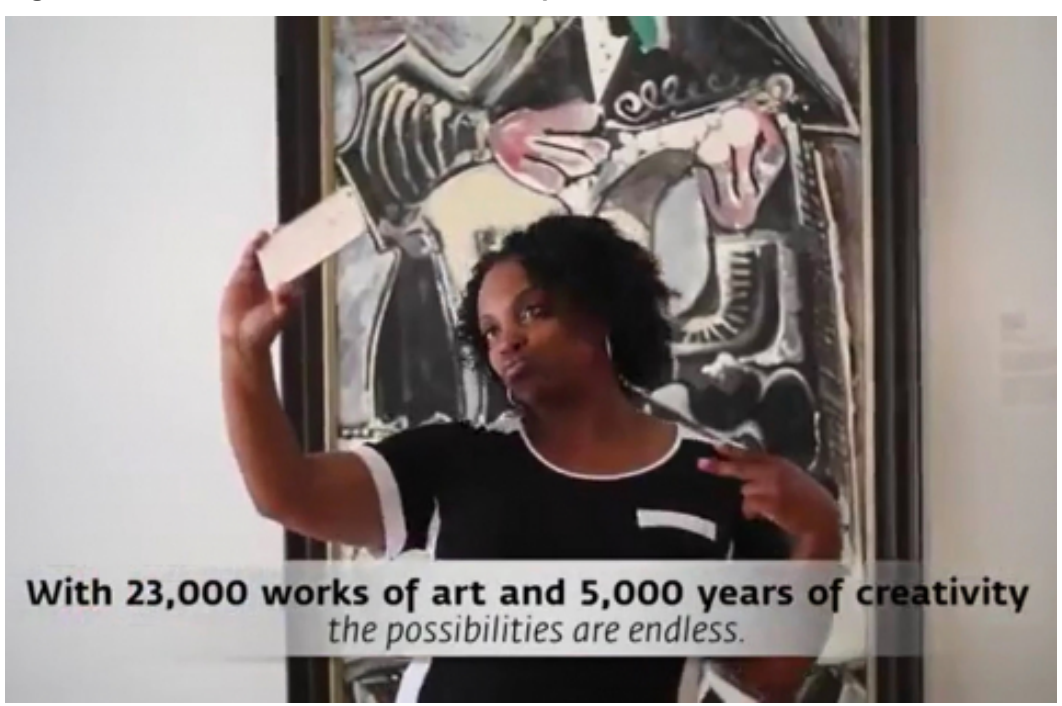

DMA App. (C) Dallas Museum of Art.

En ce sens, c'est la modification du rapport au musée qui est visée par ces vidéos en assimilant la médiation à la consommation d'un produit culturel dont l'efficacité est immédiate - disparition des médiations textuelles et d'une approche livresque de la visite, par exemple - et en combinant la reconnaissance des œuvres et du discours muséal à des pratiques culturelles dites " populaires » : jeu vidéo, photographie, etc. L'application devient alors un outil qui est représenté comme permettant de transformer le modèle classique de la visite (le bénéfice produit repose sur cette transformation). C'est de surcroît ce changement de modèle qui autorise plus globalement un nouveau rapport au musée.

\section{Paradoxe d'une métamorphose et fantasmagorie du musée}

Les vidéos promotionnelles produites pour le lancement d'une nouvelle offre de médiation par application mobile sont construites à la manière d'un tutoriel de démonstration d'un nouveau produit, mais surtout comme un guide pour une «bonne » visite muséale. Par là même, elles engagent la représentation d'un nouveau rapport au musée. Pour exprimer cette volonté sous-jacente de transformation, ces vidéos représentent d'une part un détachement de la relation au musée du seul temps de la visite - pour l'intégrer dans une temporalité élargie, plus quotidienne -, d'autre part une affiliation à des pratiques de loisir.

Une logique d'efficacité apparaît ainsi tant dans le discours lui-même que dans la visite représentée. Elle témoigne d'une négociation en cours dans les institutions muséales entre des logiques culturelles de transmission des savoirs et des logiques marchandes issues des modèles gestionnaires, ce qu'une analyse complémentaire sur les conditions de production et de diffusion des vidéos (chaîne de production, gestion des espaces de diffusion, etc.) permettrait d'approfondir. L'application mobile, tout comme le discours promotionnel l'accompagnant, illustre un processus de standardisation qui touche tant les « produits » de médiation, les formes de la visite, les visiteurs institués en public et le musée luimême. Ce processus élabore une fantasmagorie (Bonaccorsi, 2012b) et conduit à la production d'une communication muséale construisant certaines institutions en marque, dans une logique de différenciation. Les images et les représentations de la visite de musée à l'aide d'une application mobile, en étant stéréotypées, participent, du fait de leur circulation dans l'espace public, à configurer la façon 
de voir ces dispositifs et d'envisager ce que doivent être les pratiques des visiteurs. Ce travail de figuration mérite d'être pensé comme « une implication de nos pratiques » (Jeanneret, $2014: 226)$ et non comme un simple accompagnement.

La valorisation de ces nouveaux produits passe alors par la reprise des formats (vidéo courte de type tutoriel ou teaser) et registres (objet à l'infinitif, saynètes de mise en usage, forme narrative du jeu vidéo) en vigueur dans le secteur du numérique. Il en ressort une tension permanente entre une standardisation de la visite avec application tenant à la fonction de tutoriel des vidéos, et une personnalisation de l'expérience promue par les discours des industries de la communication dont se revendiquent implicitement les institutions muséales. Cette dialectique tient au travail d'équilibrisme inhérent aux discours d'escorte de ces dispositifs charriant avec eux des imaginaires sociaux, techniques et culturels du musée et du numérique (Navarro \& Renaud, 2019).

En produisant ces vidéos promotionnelles, les musées veulent montrer l'image d'une institution moderne qui est en adéquation avec les pratiques de son temps. Pour ce faire, ils convoquent et véhiculent des stéréotypes de la visite muséale, non pour les décrier mais pour montrer comment ils sont transformés par le numérique. Cette démarche tend alors de façon paradoxale - car elle repose sur une rhétorique de la différentiation - à donner du crédit aux discours circulants qui considèrent les musées de façon indifférenciée comme le lieu de la culture savante élitiste qui doit se rénover.

\section{Bibliographie}

Bonaccorsi (Julia). 2012a. "Une esthétique renouvelée de la "scène de lecture" : l’iconographie publicitaire de la lecture sur les tablettes numériques ». Mémoires du livre, [en ligne], 3(2) : https://www.erudit.org/fr/revues/memoires/2012-v3-n2-memoireso117/1009350ar/ [consulté le 5 avril 2020].

Bonaccorsi (Julia). 2012b. Fantasmagories de l'écran : Pour une approche visuelle de la textualité numérique. Mémoire d'HDR en sciences de l'information et de la communication, Celsa - Université Paris Sorbonne.

Bouquillion (Philippe), Miège (Bernard) \& Moeglin (Pierre). 2013. L'Industrialisation des biens symboliques. Les industries créatives en regard des industries culturelles. Grenoble : PUG.

Charron (Jean-Marie). 2010. « De la presse imprimée à la presse numérique. Le débat français ». Réseaux, 2(160161), p. 255-281.

Chion (Michel). 2003. Un art sonore, le cinéma. Paris : Cahiers du cinéma.

Citton (Yves). 2014. L'Économie de l'attention. Nouvel horizon du capitalisme ? Paris : La Découverte.

Dalbavie (Juliette), Da Lage (Émilie) \& Gellereau (Michèle). 2016. « Faire l'expérience de dispositifs numériques de visite et en suivre l'appropriation publique : vers de nouveaux rapports aux œuvres et aux lieux de l'expérience ? ». Études de communication, 46, p. 109-128.

Eidelman (Jacqueline), Gottesdiener (Hana) \& Le Marec (Joëlle). 2013. "Visiter les musées : expérience, appropriation, participation ». Culture \& Musées, hors-série, « La muséologie : 20 ans de recherches », p. 73-113.

Escande-Gauquié (Pauline) \& Jeanne-Perrier (Valérie). 2017. « Le partage photographique : le régime performatif de la photo ». Communication \& Langages, 194(4), p. 21-27.

Gellereau (Michèle). 2005. Les Mises en scène de la visite guidée. Communication et médiation. Paris : L'Harmattan (Communication et civilisation).

Ibekwe-Sanjuan (Fidelia) \& Paquienseguy (Françoise). 2015. " Open, Big, Collaboration : trois utopies de l'innovation au xxie siècle », p. 15-29 in Big Data - Open Data : Quelles valeurs ? Quels enjeux? / sous la direction d'Evelyne Broudoux. Louvain-la-Neuve : De Boeck Supérieur.

Jacobi (Daniel) \& Le Roy (Maryline). 2013. La Signalétique patrimoniale. Principes et mise en ouvre. Arles : Éditions Errance.

Jacobi (Daniel). 2016. Textexpo : Produire, éditer et afficher des textes d'exposition. Dijon : OCIM.

Jeanneret (Yves). 2014. Critique de la trivialité. La médiation de la communication, enjeu de pouvoir. Paris : Éditions Non Standard.

Jeanneret (Yves), Lallement (Emmanuelle) \& Menrath (Joëlle) (dir.). 2005. Le Téléphone mobile aujourd'hui : Usages, représentations et comportements sociaux. Rapport de recherche pour l'AFOM.

Jeanneret (Yves) \& Souchier (Emmanuel). 1999. « Pour une poétique de "l'écrit d'écran” ». Xoana, 6-7, p. 97-107.

Jeanneret (Yves), Souchier (Emmanuel) \& Le Marec (Joëlle) (dir.). 2003 Lire, écrire, récrire. Objets, signes et pratiques des médias informatisés. Paris : Bibliothèque publique d’information.

Lesaffre (Gaëlle), Watremez (Anne) \& Flon (Émilie). 2014. « Les applications mobiles de musées et de sites patrimoniaux en France, quelles propositions de médiation? ». La Lettre de l'OCIM, 154, p. 5-13.

Martin-Juchat (Fabienne) \& Staii (Adrian). 2016. L'Industrialisation des émotions : vers une radicalisation de la modernité. Paris : L'Harmattan.

Navarro (Nicolas) \& Renaud (Lise). 2019. "La médiation numérique au musée en procès ». Revue française des Sciences de l'information et de la communication [en ligne], 16 : https://journals.openedition.org/rfsic/5592 [consulté le 14 avril 2020].

Péninou (Georges). 1972. L'Intelligence de la publicité. Paris : Robert Laffont.

Renaud (Lise). 2016. " L'imaginaire publicitaire des techniques info-communicationnelles : vers la technicisation des émotions ", p. 65-89 in L'Industrialisation des émotions. Vers une radicalisation de la modernité ? / sous la direction d'Adrian Staii et Fabienne Martin-Juchat. Paris : L’Harmattan.

Schmitt (Daniel) \& Meyer-Chemenska (Muriel). 2015. "Vingt ans de numérique dans les musées : entre monstration et effacement ». La Lettre de l'OCIM, 162, p. 53-57.

Regourd (Martine) (dir.). 2012. Musées en mutation. Un espace public à revisiter. Paris : L'Harmattan (Gestion de la culture).

Tardy (Cécile). 2015. " La médiation d'authenticité des substituts numériques » in Mémoire et nouveaux patrimoines [en ligne] / sous la direction de Cécile Tardy et Vera Dodebei. Marseille : OpenEdition Press : 
https://books.openedition.org/oep/453 [consulté le14 avril 2020].

DOI : $10.4000 / 1895.2$

Tobelem (Jean-Michel) (dir.). 2010. Le Nouvel Age des musées. Paris : Armand Colin.

Veron (Éliséo). 1991. «Pour en finir avec la "communication” ». Réseaux, 9(46-47), p. 119-126.

DOI : $10.3406 /$ reso.1991.1835

\section{Annexe}

Liste des vidéos du corpus (pages suivantes)

\begin{tabular}{|c|c|c|c|c|c|c|c|c|}
\hline$N^{\circ}$ & $\begin{array}{l}\text { Nom de } \\
\text { l'application }\end{array}$ & $\begin{array}{l}\text { Année de } \\
\text { lancement }\end{array}$ & $\begin{array}{l}\text { Nom du } \\
\text { musée }\end{array}$ & $\begin{array}{l}\text { Catégorie } \\
\text { du musée }\end{array}$ & $\begin{array}{l}\text { Titre de la } \\
\text { vidéo }\end{array}$ & Consulté en avril 2020 sur : & Format & $\begin{array}{l}\text { Producteurs } \\
\text { de } \\
\text { l'application }\end{array}$ \\
\hline 1 & $\begin{array}{l}\text { Louvre : ma } \\
\text { visite }\end{array}$ & 2016 & $\begin{array}{l}\text { Musée du } \\
\text { Louvre (Paris) }\end{array}$ & $\begin{array}{l}\text { Musée } \\
\text { d'art }\end{array}$ & \begin{tabular}{|l} 
Teaser \\
Louvre : ma \\
visite
\end{tabular} & https://www.louvre.fr/louvre-ma-visite & $15 \mathrm{sec}$ & $\begin{array}{l}\text { Droits Musée } \\
\text { du Louvre }\end{array}$ \\
\hline 2 & $\begin{array}{l}\text { Musée d'arts } \\
\text { de Nantes }\end{array}$ & 2017 & $\begin{array}{l}\text { Musée d'arts } \\
\text { de Nantes }\end{array}$ & $\begin{array}{l}\text { Musée } \\
\text { d'art }\end{array}$ & \begin{tabular}{|l|} 
L'application \\
MA VISITE \\
du Musée \\
d'arts de \\
Nantes
\end{tabular} & $\begin{array}{l}\text { https://museedartsdenantes.nantesmetropole.fr/ } \\
\text { mavisite }\end{array}$ & $\begin{array}{l}1 \mathrm{~min} \\
33 \mathrm{sec}\end{array}$ & $\begin{array}{l}\text { Nantes } \\
\text { Métropole }\end{array}$ \\
\hline 3 & Arkéofil & 2018 & $\begin{array}{l}\text { Musée } \\
\text { départemental } \\
\text { Arles antique }\end{array}$ & $\begin{array}{l}\text { Musée } \\
\text { d'histoire } \\
\text { et de } \\
\text { société }\end{array}$ & $\begin{array}{l}\text { Teaser 1: } \\
\text { Arkéofil }\end{array}$ & $\begin{array}{l}\text { https://www.youtube.com/watch? } \\
\text { v=kfwURO7CqYM }\end{array}$ & $\begin{array}{l}1 \mathrm{~min} \\
10 \mathrm{sec}\end{array}$ & MDAA \\
\hline 4 & \begin{tabular}{|l} 
Visite \\
Museum \\
Mobile
\end{tabular} & 2018 & $\begin{array}{l}\text { Muséum de } \\
\text { Toulouse }\end{array}$ & $\begin{array}{l}\text { Musée de } \\
\text { sciences } \\
\text { et } \\
\text { techniques }\end{array}$ & $\begin{array}{l}\text { Une nouvelle } \\
\text { façon de } \\
\text { visiter le } \\
\text { Muséum de } \\
\text { Toulouse }\end{array}$ & $\begin{array}{l}\text { https://www.museum.toulouse.fr/visite-museum- } \\
\text { mobile }\end{array}$ & $\begin{array}{l}2 \mathrm{~min} \\
9 \mathrm{sec}\end{array}$ & $\begin{array}{l}\text { Production : } \\
\text { Muséum de } \\
\text { Toulouse / } \\
\text { Toulouse } \\
\text { Métropole } \\
\text { Agence de } \\
\text { réalisation } \\
\text { vidéo: } \\
\text { Pinkanova }\end{array}$ \\
\hline 5 & $\begin{array}{l}\text { Allen } \\
\text { enquête au } \\
\text { Musée de } \\
\text { I'homme }\end{array}$ & 2016 & $\begin{array}{l}\text { Musée de } \\
\text { l'homme } \\
\text { (Paris) }\end{array}$ & $\begin{array}{l}\text { Musée de } \\
\text { sciences } \\
\text { et } \\
\text { techniques }\end{array}$ & \begin{tabular}{|l|} 
Application \\
mobile : Allen \\
mène \\
l'enquête au \\
Musée de \\
l'homme
\end{tabular} & $\begin{array}{l}\text { http://www.museedelhomme.fr/fr/media-video/ } \\
4090\end{array}$ & $54 \mathrm{sec}$ & $\begin{array}{l}\text { Museum } \\
\text { d'histoire } \\
\text { naturelle } \\
+ \text { Orange }\end{array}$ \\
\hline 6 & Grand Palais & 2014 & $\begin{array}{l}\text { Grand Palais } \\
\text { (Paris) }\end{array}$ & $\begin{array}{l}\text { Musée } \\
\text { d'art }\end{array}$ & \begin{tabular}{|l|} 
Grand \\
Palais / \\
L'application \\
de \\
l'exposition
\end{tabular} & https://www.dailymotion.com/video/x28289w & $\begin{array}{l}1 \mathrm{~min} \\
23 \mathrm{sec}\end{array}$ & $\begin{array}{l}\text { RMN - Granc } \\
\text { Palais } \\
\text { + Mazedia }\end{array}$ \\
\hline 7 & $\begin{array}{l}\text { Musée } \\
\text { Rodin }\end{array}$ & 2016 & $\begin{array}{l}\text { Musée Rodin } \\
\text { (Paris) }\end{array}$ & $\begin{array}{l}\text { Musée } \\
\text { d'art }\end{array}$ & \begin{tabular}{|l|} 
Musée \\
Rodin : \\
découvrez le \\
guide \\
multimédia
\end{tabular} & https://www.dailymotion.com/video/x3i6g3s & $38 \mathrm{sec}$ & $\begin{array}{l}\text { Museum } \\
\text { Guides Ltd }\end{array}$ \\
\hline 8 & $\begin{array}{l}\text { MBA, Musée } \\
\text { des beaux- } \\
\text { arts de Lyon }\end{array}$ & 2011 & $\begin{array}{l}\text { Musée des } \\
\text { beaux-arts de } \\
\text { Lyon }\end{array}$ & $\begin{array}{l}\text { Musée } \\
\text { d'art }\end{array}$ & \begin{tabular}{|l|} 
Musée des \\
beaux-arts \\
de Lyon : \\
application \\
gratuite / \\
Free apps
\end{tabular} & $\begin{array}{l}\text { https://www.youtube.com/watch? } \\
\text { v=c08KmevW6wY }\end{array}$ & $\begin{array}{l}1 \mathrm{~min} \\
21 \mathrm{sec}\end{array}$ & $\begin{array}{l}\text { My Lucky Da } \\
+ \text { MBA Lyon }\end{array}$ \\
\hline 9 & $\begin{array}{l}\text { Incroyable } \\
\text { Grévin }\end{array}$ & 2016 & $\begin{array}{l}\text { Musée Grévin } \\
\text { (Paris) }\end{array}$ & $\begin{array}{l}\text { Musée } \\
\text { d'histoire } \\
\text { et de } \\
\text { société }\end{array}$ & \begin{tabular}{|l|} 
Découvrez \\
l'application \\
« Grévin \\
Paris! » - \\
Teaser
\end{tabular} & $\begin{array}{l}\text { https://www.youtube.com/watch? } \\
\text { v=050dIQRC1IQ }\end{array}$ & $35 \mathrm{sec}$ & $\begin{array}{l}\text { Musée } \\
\text { Grévin, } \\
\text { smArtapps }\end{array}$ \\
\hline 10 & HistoPad & 2018 & $\begin{array}{l}\text { Airborne } \\
\text { Museum } \\
\text { (Sainte-Mère- } \\
\text { Église) }\end{array}$ & \begin{tabular}{|l} 
Musée \\
d'histoire \\
et de \\
société
\end{tabular} & & $\begin{array}{l}\text { https://www.airborne-museum.org/ } \\
\text { airbornereality-4/ }\end{array}$ & $\begin{array}{l}1 \mathrm{~min} \\
6 \mathrm{sec}\end{array}$ & $\begin{array}{l}\text { Airborne } \\
\text { Museum } \\
\text { Histovery }\end{array}$ \\
\hline 11 & RIJKS & \begin{tabular}{|l}
2018 \\
(nouvelle \\
version)
\end{tabular} & $\begin{array}{l}\text { Rijksmuseum } \\
\text { (Amsterdam) }\end{array}$ & $\begin{array}{l}\text { Musée } \\
\text { d'art }\end{array}$ & \begin{tabular}{|l|} 
New Rijks \\
App - The \\
Rijksmuseum \\
in your \\
pocket
\end{tabular} & $\begin{array}{l}\text { https://www.youtube.com/watch? } \\
\text { v=F8tzNPvwwAE }\end{array}$ & $\begin{array}{l}1 \mathrm{~min} \\
5 \mathrm{sec}\end{array}$ & Rijksmuseur \\
\hline & & & & & & & & \\
\hline
\end{tabular}




\begin{tabular}{|c|c|c|c|c|c|c|c|c|}
\hline \multirow[t]{2}{*}{12} & \multirow[t]{2}{*}{ MANN App } & \multirow[t]{2}{*}{2016} & $\begin{array}{l}\text { Musée } \\
\text { d'archéologie } \\
\text { nationale de } \\
\text { Naples }\end{array}$ & $\begin{array}{l}\text { Musée } \\
\text { d'histoire } \\
\text { et de } \\
\text { société }\end{array}$ & $\begin{array}{l}\text { MANN App } \\
\text { Pompeii A/R }\end{array}$ & $\begin{array}{l}\text { https://www.museoarcheologiconapoli.it/en/ } \\
\text { mann-app/ }\end{array}$ & $57 \mathrm{sec}$ & MANN \\
\hline & & & \multirow[b]{2}{*}{$\begin{array}{l}\text { National } \\
\text { Military } \\
\text { Museum } \\
\text { (Soesterberg) }\end{array}$} & \multirow[b]{2}{*}{$\begin{array}{l}\text { Musée } \\
\text { d'histoire } \\
\text { et de } \\
\text { société }\end{array}$} & \multirow[b]{2}{*}{$\begin{array}{l}\text { Operatie } \\
\text { «New } \\
\text { Dawn » in } \\
\text { het Nationaal } \\
\text { Militair } \\
\text { Museum }\end{array}$} & & & \\
\hline 13 & New Dawn & $\begin{array}{l}2019 \\
\text { (nouvelle } \\
\text { version) }\end{array}$ & & & & $\begin{array}{l}\text { https://www.youtube.com/watch? } \\
\text { v=n2PmstzYUEY }\end{array}$ & $\begin{array}{l}1 \mathrm{~min} \\
8 \mathrm{sec}\end{array}$ & NMM \\
\hline 14 & Mon MCQ & 2018 & $\begin{array}{l}\text { Musée de la } \\
\text { civilisation de } \\
\text { Québec }\end{array}$ & $\begin{array}{l}\text { Musée } \\
\text { d'histoire } \\
\text { et de } \\
\text { société }\end{array}$ & $\begin{array}{l}\text { Mon MCQ : } \\
\text { Tutoriel }\end{array}$ & $\begin{array}{l}\text { https://www.mcq.org/en/produit-numerique? } \\
\text { id=736583 }\end{array}$ & $\begin{array}{l}2 \min \\
21 \mathrm{sec}\end{array}$ & $\begin{array}{l}\text { Musée de la } \\
\text { civilisation } \\
+ \text { Cortex + lg }\end{array}$ \\
\hline 15 & $\begin{array}{l}\text { Quelle } \\
\text { histoire }\end{array}$ & 2017 & $\begin{array}{l}\text { Musée des } \\
\text { beaux-arts de } \\
\text { Montréal }\end{array}$ & $\begin{array}{l}\text { Musée } \\
\text { d'art }\end{array}$ & $\begin{array}{l}\text { Quelle } \\
\text { histoire ! } \\
\text { What a } \\
\text { History! }\end{array}$ & $\begin{array}{l}\text { https://www.facebook.com/mbamtl/videos/ } \\
\text { quelle-histoire-what-a-history/ } \\
\text { 10160083511540235/ }\end{array}$ & $\begin{array}{l}1 \mathrm{~min} \\
19 \mathrm{sec}\end{array}$ & $\begin{array}{l}\text { Musée des } \\
\text { beaux-arts dt } \\
\text { Montréal } \\
+ \text { Ubisoft }\end{array}$ \\
\hline 16 & $\begin{array}{l}\text { The } \\
\text { Guggenheim } \\
\text { Museum }\end{array}$ & $\begin{array}{l}2015 \text { (ajout } \\
\text { fonctionnalité } \\
\text { Near me) }\end{array}$ & $\begin{array}{l}\text { Guggenheim } \\
\text { Museum (New } \\
\text { York) }\end{array}$ & $\begin{array}{l}\text { Musée } \\
\text { d'art }\end{array}$ & $\begin{array}{l}\text { Introducing } \\
\text { Near Me on } \\
\text { the } \\
\text { Guggenheim } \\
\text { App }\end{array}$ & $\begin{array}{l}\text { https://twitter.com/guggenheim/status/ } \\
677214966735175680\end{array}$ & $\begin{array}{l}1 \mathrm{~min} \\
2 \mathrm{sec}\end{array}$ & $\begin{array}{l}\text { Guggenheim } \\
+ \text { Fondation } \\
\text { Blommberg }\end{array}$ \\
\hline 17 & $\begin{array}{l}\text { SFMOMA } \\
\text { Audio }\end{array}$ & 2018 & $\begin{array}{l}\text { San Francisco } \\
\text { Museum of } \\
\text { Modern Art }\end{array}$ & $\begin{array}{l}\text { Musée } \\
\text { d'art }\end{array}$ & $\begin{array}{l}\text { An App That } \\
\text { Will Forever } \\
\text { Change How } \\
\text { You Enjoy } \\
\text { Museums }\end{array}$ & $\begin{array}{l}\text { https://mw17.mwconf.org/glami/the-sfmoma- } \\
\text { app/ }\end{array}$ & $\begin{array}{l}3 \mathrm{~min} \\
27 \mathrm{sec}\end{array}$ & $\begin{array}{l}\text { Blommberg } \\
\text { Philanthropie } \\
+ \text { Cuseum }\end{array}$ \\
\hline 18 & Lacma & $\begin{array}{l}2015 \\
\text { (nouvelle } \\
\text { version) }\end{array}$ & $\begin{array}{l}\text { Los Angeles } \\
\text { County } \\
\text { Museum of } \\
\text { Art }\end{array}$ & $\begin{array}{l}\text { Musée } \\
\text { d'art }\end{array}$ & $\begin{array}{l}\text { Lacma's new } \\
\text { mobile app }\end{array}$ & http://www.lacma.org/mobile & $55 \mathrm{sec}$ & Lacma \\
\hline 19 & $\begin{array}{l}\text { The Frick } \\
\text { Collection }\end{array}$ & 2014 & $\begin{array}{l}\text { The Frick } \\
\text { Collection } \\
\text { (New York) }\end{array}$ & $\begin{array}{l}\text { Musée } \\
\text { d'art }\end{array}$ & $\begin{array}{l}\text { The Frick } \\
\text { Collection } \\
\text { App }\end{array}$ & https://www.frick.org/interact/app & $\begin{array}{l}1 \mathrm{~min} \\
6 \mathrm{sec}\end{array}$ & $\begin{array}{l}\text { Stevens } \\
\text { Institute of } \\
\text { Technology } \\
+ \text { the Frick } \\
\text { Collection } \\
\text { + financemer } \\
\text { Sandeep } \\
\text { Mathrani }\end{array}$ \\
\hline 20 & $\begin{array}{l}\text { DMA, Dallas } \\
\text { Museum of } \\
\text { Art }\end{array}$ & $\begin{array}{l}2017 \\
\text { (nouvelle } \\
\text { version) }\end{array}$ & $\begin{array}{l}\text { Dallas } \\
\text { Museum of } \\
\text { Art }\end{array}$ & $\begin{array}{l}\text { Musée } \\
\text { d'art }\end{array}$ & $\begin{array}{l}\text { The DMA } \\
\text { App }\end{array}$ & https://www.dma.org/DMAapp & $\begin{array}{l}1 \mathrm{~min} \\
10 \mathrm{sec}\end{array}$ & DMA \\
\hline 21 & & 2015 & $\begin{array}{l}\text { Canadian } \\
\text { Museum for } \\
\text { Human Rights } \\
\text { (Winnipeg) }\end{array}$ & $\begin{array}{l}\text { Musée } \\
\text { d'histoire } \\
\text { et de } \\
\text { société }\end{array}$ & & $\begin{array}{l}\text { https://humanrights.ca/interactive-awards/ } \\
\text { mobile-app }\end{array}$ & $\begin{array}{l}4 \mathrm{~min} \\
47 \mathrm{sec}\end{array}$ & CMHR \\
\hline 22 & The Met & 2014 & $\begin{array}{l}\text { Metropolitan } \\
\text { Museum (New } \\
\text { York) }\end{array}$ & $\begin{array}{l}\text { Musée } \\
\text { d'art }\end{array}$ & $\begin{array}{l}\text { The MET } \\
\text { App }\end{array}$ & https://vimeo.com/107533738 & $58 \mathrm{sec}$ & $\begin{array}{l}\text { Agence de } \\
\text { pub } \\
\text { numérique : } \\
\text { Dép. } \\
\text { Numérique d } \\
\text { MET } \\
+ \text { Instrument } \\
\text { (agence } \\
\text { numérique dt } \\
\text { Portland) } \\
\text { Bloomberg } \\
\text { Philanthropie }\end{array}$ \\
\hline 23 & & 2018 & $\begin{array}{l}\text { Science } \\
\text { Museum } \\
\text { Londres }\end{array}$ & $\begin{array}{l}\text { Musée de } \\
\text { sciences } \\
\text { et } \\
\text { techniques }\end{array}$ & $\begin{array}{l}\text { Treasure } \\
\text { Hunters app } \\
\text { trailer }\end{array}$ & $\begin{array}{l}\text { https://learning- } \\
\text { resources.sciencemuseum.org.uk/resources/ } \\
\text { treasure-hunters-gamify-museum-visit/ }\end{array}$ & $\begin{array}{l}1 \mathrm{~min} \\
12 \mathrm{sec}\end{array}$ & $\begin{array}{l}\text { Science } \\
\text { Museum } \\
\text { (SCMG } \\
\text { Enterprises) }\end{array}$ \\
\hline
\end{tabular}

\section{Notes}

1 Il s'agit de logiciels informatiques consultables sur tablette ou téléphone portable et accessibles directement sur place ou via un téléchargement. Le Club Innovation \& Culture France (CLIC France) propose sur son site Internet un panorama des applications mobiles disponibles en France : http://www.club-innovation-culture.fr/applicationsmobiles-france-3/ [consulté le 3 avril 2020]. Celui-ci présente l'application pour l'exposition Bruegel, Memling, Van Eyck du Musée Jacquemart-André en 2009 comme la première ayant existé en France. 
2 Les applications (détaillées en annexe) sont celles du Musée du Louvre (Paris), Musée d'arts de Nantes, Musée départemental Arles antique, Muséum de Toulouse, Musée de l'homme (Paris), Grand Palais (Paris), Musée Rodin (Paris), Musée des beaux-arts de Lyon, Musée Grévin (Paris), Airborne Museum (Sainte-Mère-Église), Rijksmuseum (Amsterdam), Musée d'archéologie nationale de Naples, National Military Museum (Soesterberg, Pays-Bas), Musée de la civilisation de Québec, Musée des beaux-arts de Montréal, Guggenheim Museum (New York), San Francisco Museum of Modern Art, Los Angeles County Museum of Art, The Frick Collection (New York), Dallas Museum of Art, Canadian Museum for Human Rights (Winnipeg), Metropolitan Museum (New York), Science Museum Londres.

3 « Musée des beaux-arts de Lyon : application gratuite / Free apps », « The DMA App » ou « The Frick Collection App ».

4 Le terme de signe passeur est une notion élaborée dans le cadre de la théorie des écrits d'écran pour désigner les liens hypertextes en les appréhendant dans leur fonction sémiotique et non seulement dans leur fonction instrumentale (Jeanneret \& Souchier, 1999 ; Jeanneret et al., 2003).

5 Par exemple, les sites de la revue Wired ou de MuseWeb.

6 Musée Rodin, Musée de la civilisation de Québec, Grand Palais, Musée du Louvre.

7 À titre d'exemples : "Download The Frick Collection's app for access to information on more than 1,ooo works of art in the museum's collection " (The Frick Collection) ; "With 23,00o works of art and 5,00o years of creativity, the possibilities are endless » (Dallas Museum of Art).

8 "You know what else is very useful? Now, you always know exactly where you are in the museum " (Rijksmuseum).

9 Guggenheim New York, Rijksmuseum, MET, DMA, MBA Lyon, The Frick Collection, Musée d'arts de Nantes, Muséum de Toulouse, SFMOMA.

10 C'est le cas de la vidéo du Musée archéologique national de Naples où est représentée une galerie de sculptures blanches, comme stéréotype du musée d'archéologie.

11 À l'exception de la vidéo du Musée départemental Arles antique.

12 La vidéo du Human Rights Museum met en scène un visiteur non voyant déambulant à l'aide d'un marquage au sol en $3 \mathrm{D}$ et d'une numérotation en braille des textes de l'exposition suivie d'une portion de vidéo en langage des signes.

13 «You can play at any of our museums or even at home » (Science Museum).

14 "Save your favorite works to enjoy offline and share via email, Facebook, Twitter, Pinterest, and Google+ » (The Frick Collection).

15 «You can now explore the museum yourself» (Rijksmuseum).

16 Le poids de la nature de la collection muséale semble appuyer ce constat tant apparait centrale dans les musées de beaux-arts une reconnaissance individualisée des œuvres, alors que dans les musées de science et d'histoire, la discussion autour du discours muséal est plus évoquée, comme l'explicite le cas du Science Museum : "Get together with your family and friends to create a museum tour that's different every time you play. "

17 « Partagez vos coups de cœur » (MBA Lyon), « Get social! Follow the DMA right from the app and share your favorites » (DMA), « Partagez vos photos sur les réseaux sociaux » (Grand Palais).

18 « Shake it up! Let the DMA app randomly select art for you » (DMA).

\section{Table des illustrations}

Titre Figure 1. Présentation sous forme d'objet à l'infinitif.

Crédits Application Musée Rodin. @ Musée Rodin.

URI http://journals.openedition.org/culturemusees/docannexe/image/4713/img-1.jpg

Fichier image/jpeg, 132k

Titre Figure 2. Saynète de mise en usage.

Crédits Application The MET. @ Metropolitan Museum of Art.

URL http://journals.openedition.org/culturemusees/docannexe/image/4713/img-2.jpg

Fichier image/jpeg, 272k

Titre Figure 3. Représentation d'une relation individuelle et exclusive. Lacma Mobile App.

Fil. Crédits @ Los Angeles County Museum of Art.

II URI http://journals.openedition.org/culturemusees/docannexe/image/4713/img-3.jpg

Fichier image/jpeg, 232k

Titre Figure 4. Représentation d'une relation individuelle et exclusive. Application Musée d'arts de Nantes.

Crédits $\odot$ Musée d'arts de Nantes.

URI http://journals.openedition.org/culturemusees/docannexe/image/4713/img-4.jpg

Fichier image/jpeg, 144k

Titre Figure 5. Préparatif d'avant-visite.

Crédits Mann App. ( ) Musée d'archéologie nationale de Naples.

URL http://journals.openedition.org/culturemusees/docannexe/image/4713/img-5.jpg

Fichier image/jpeg, 220k

Titre Figure 6. Prolongation d'après-visite.

Crédits Visite museum Mobile. ( ) Muséum de Toulouse.

URI http://journals.openedition.org/culturemusees/docannexe/image/4713/img-6.jpg

Fichier image/jpeg, 176k

Titre Figure 7. Mise en scène d'une visiteuse parlant aux objets. 
Crédits Rijksmuseum App. @ Rijksmuseum.

URI http://journals.openedition.org/culturemusees/docannexe/image/4713/img-7.jpg

Fichier image/jpeg, 256k

Titre Figure 8. Mise en scène d'une visiteuse se prenant en selfie devant une œuvre.

Crédits DMA App. ( ) Dallas Museum of Art.

URL http://journals.openedition.org/culturemusees/docannexe/image/4713/img-8.jpg

Fichier image/jpeg, 188k

\section{Pour citer cet article}

Référence papier

Nicolas Navarro et Lise Renaud, «Fantasmagorie du musée : vers une visite numérique et récréative », Culture \& Musées, 35 | 2020, 133-163.

Référence électronique

Nicolas Navarro et Lise Renaud, «Fantasmagorie du musée : vers une visite numérique et récréative », Culture \& Musées [En ligne], 35 | 2020, mis en ligne le 01 juin 2020, consulté le 31 mai 2021. URL:

http://journals.openedition.org/culturemusees/4713; DOI : https://doi.org/10.4000/culturemusees.4713

\section{Auteurs}

\section{Nicolas Navarro}

Université Lyon 2, Elico

Nicolas Navarro est maître de conférences en sciences de l'information et de la communication à l'Université Lumière Lyon 2 et membre d'Elico (EA 4147). Ses travaux portent sur la mise en communication des territoires à travers une approche ethno-sémiotique des objets et êtres culturels, et de leurs médiations, en particulier du patrimoine et des musées. Ses dernières publications portent ainsi sur une analyse critique des discours d'escorte autour des projets numériques patrimoniaux. En 2019, il a publié : avec Nolwenn Pianezza et Lise Renaud, « Pour une archéologie de l'injonction : leitmotivs dans la presse autour des projets numériques patrimoniaux », dans la revue en ligne Les Enjeux de l'information et de la communication ; avec Lise Renaud « La médiation numérique au musée en procès ", dans la revue française des sciences de l'information et de la communication ; "Le patrimoine par l'image : socio-sémiotique des centres d'interprétation de l'architecture et du patrimoine » dans le $\mathrm{n}^{\circ} 36$ de la revue Figures de l'art.

Courriel : n.navarro[at]univ-lyon2.fr

\section{Articles du même auteur}

Le patrimoine métamorphe. Circulation et médiation du patrimoine urbain dans les Villes et pays d'art et d'histoire [Texte intégral]

Paru dans Culture \& Musées, $31 \mid 2018$

Françoise Lempereur (dir.). 2017. Patrimoine culturel immatériel [Texte intégral]

Paru dans Culture \& Musées, $31 \mid 2018$

Politiques patrimoniales et touristiques des territoires : Les centres d'interprétation de l'architecture et du patrimoine [Texte intégral]

Heritage and Tourism Policies of the Territories: The Interpretation Centers for Architecture and Heritage

Políticas patrimoniales y turísticas de los territorios : Los centros de interpretación de la Arquitectura y del Patrimonio

Paru dans Culture \& Musées, $23 \mid 2014$

Lise Renaud

Avignon Université, Centre Norbert Elias

Lise Renaud est maîtresse de conférences en sciences de l'information et de la communication à Avignon

Université et membre du Centre Norbert Elias (UMR 8562). Ses recherches portent sur les relations entre discours d'escorte, imaginaires et pratiques socioculturelles, notamment celles appareillées de dispositifs techniques mobiles. Adoptant une approche socio-sémiotique, ses travaux visent à qualifier les processus de médiatisations, d'inscriptions et de figurations visuelles à l'œuvre dans les relations que nous tissons avec les médias informatisés. Ses dernières publications portent sur le rôle des figures et valeurs associées au numérique dans les pratiques patrimoniales. Parmi ses publications récentes, on peut citer « Le paratexte pour penser la configuration des pratiques numériques ", dans Communication \& Langages, et « La médiation numérique au musée en procès », écrit avec Nicolas Navarro, dans la revue en ligne RFSIC (2019).

Courriel : lise.renaud[at]univ-avignon.fr

\section{Droits d'auteur}

Culture \& Musées 